\title{
Quantum Trajectories for Realistic Detection
}

\author{
P. Warszawski ${ }^{1}$, H.M. Wiseman ${ }^{1,2}$, and H. Mabuchi ${ }^{2}$ \\ 1 School of Science, Griffith University, Nathan, Brisbane, Queensland 4111 Australia \\ ${ }^{2}$ Norman Bridge Laboratory of Physics 12-33, California Institute of Technology, Pasadena, CA 91125, USA
}

(February 1, 2008)

\begin{abstract}
Quantum trajectories describe the stochastic evolution of an open quantum system conditioned on continuous monitoring of its output, such as by an ideal photodetector. Here we derive (nonMarkovian) quantum trajectories for realistic photodetection, including the effects of efficiency, dead time, bandwidth, electronic noise, and dark counts. We apply our theory to a realistic cavity QED scenario and investigate the impact of such detector imperfections on the conditional evolution of the system state. A practical theory of quantum trajectories with realistic detection will be essential for experimental and technological applications of quantum feedback in many areas.
\end{abstract}

03.65.Yz, 03.65.Ta, 42.50.Lc, 42.50.Ar

\section{INTRODUCTION}

The limited utility of quantum measurement theory as axiomatized by von Neumann [1] for describing practical laboratory measurements has necessitated the development of more general measurement theories [2,3]. In the past decade the application of such theories has become widespread in quantum optics, in particular for describing continuous monitoring of the photoemission from radiatively damped open systems. They describe the evolution of the conditioned system state in terms of quantum jumps 沺 6] for direct detection and quantum diffusion [6.7] for dyne detection. The stochastic evolution equation, termed a quantum trajectory, has also been applied in mesoscopic electronics [B].

Thus far, the main practical utility of quantum trajectory theory has been in improving the computational efficiency of simulations used to compare models with experimental data. But it is now gaining increasing importance as the quantum generalization of Kalman filtering, which provides essential signal-processing methods in classical estimation, communication, and control engineering. Quantum trajectory theory should in principle play the same pivotal role for emerging quantum analogs of these technologies [9 11]. Before this can happen it is essential that the theory be extended to account for the imperfections of realistic measurement devices, as non-ideal detector dynamics can dramatically affect the proper inference from measured signals to the conditional quantum state of an observed system.

In this paper we present the theory of quantum trajectories for realistic photodetection. We model both photon counters and photoreceivers (for homodyne detection) and include the effects of efficiency, dead time, bandwidth, electronic noise, and dark counts. The proper treatment of bandwidth limitations and electronic noise are of particular significance as these imperfections are inevitable and predominant concerns in any practical context. They are of central importance in the current generation of experiments on quantum-limited measurement in atomic [12] and condensed matter [13] systems.

Our theory works by embedding the system within a supersystem that obeys a Markovian equation. If the set of (classical) detector states is $\mathbf{S}$, then the supersystem is described by the set $\left\{\rho_{s}: s \in \mathbf{S}\right\}$. Here $\operatorname{Tr}\left[\rho_{s}\right]$ is the probability that the apparatus is in state $s$, and $\rho_{s} / \operatorname{Tr}\left[\rho_{s}\right]$ is the system state given this event.

\section{THE SYSTEM}

In this paper we take the monitored system to be a twolevel atom (TLA), classically driven at Rabi frequency $\Omega$ and radiatively damped at rate $\Gamma$. The TLA obeys the unconditional master equation (ME)

$$
\dot{\rho}=\mathcal{L} \rho=-i(\Omega / 2)\left[\sigma_{x}, \rho\right]+\Gamma\left(\sigma \rho \sigma^{\dagger}-\frac{1}{2}\left\{\sigma^{\dagger} \sigma, \rho\right\}\right),
$$

where $\sigma$ is the atomic lowering operator, and $\sigma_{x}=\sigma+\sigma^{\dagger}$. Time arguments are not included unless they are necessary for the reader's understanding. In reality, it is difficult to detect a significant fraction of an atom's fluorescence. However, the ME (11) also describes, in a suitable regime [14], the damping of an atom through a cavity mode. This produces an easily detectable output beam. In this scenario, the effective decay rate $\Gamma$ may be much larger than that of a bare atom, and we have this in mind when choosing $\Gamma=300 \mathrm{Ms}^{-1}$ for our simulations.

\section{PHOTON COUNTER}

An avalanche photodiode (APD) operating in Geiger mode produces a macroscopic current pulse in response to an incident photon. It consists of a p-n junction operated under a reverse bias greater than the breakdown voltage 15]. Under these conditions we can describe the diode by just three classical states (see Fig. 1). The first (0) is a stable low-current state in which there are no charge carriers in the depletion region of the junction. 
The transition from 0 to the second state (1) takes place when an electron-hole pair is created in the depletion region by an incident photon (with quantum efficiency $\eta$ ) or by thermally initiated 'dark counts' occurring at a rate $\gamma_{\mathrm{dk}}$. Further impact ionization leads to an avalanche, until the current reaches some threshold value and a detection is registered, thus changing the state of the APD to 2 . The transition from 1 to 2 has a random duration (we assume Poissonian) with mean $\gamma_{\mathrm{r}}^{-1}$ (the 'response time'). The avalanche is then arrested by the application of a negative-going voltage pulse that temporarily brings the bias voltage below the breakdown value 17]. This results in a fixed 'dead time', $\tau_{\mathrm{dd}}$, during which the APD cannot detect photons, after which it is restored to state 0.

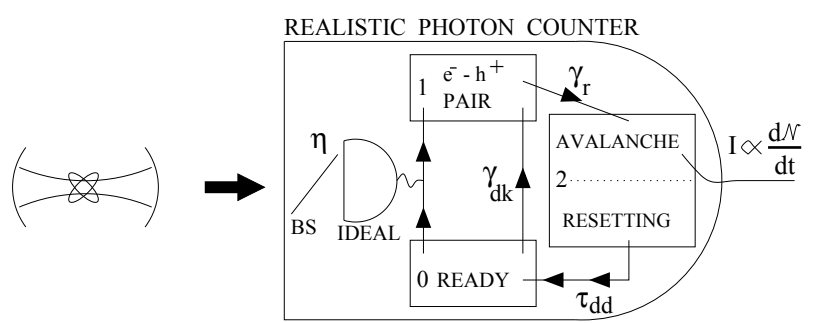

FIG. 1. Realistic photon counting by an avalanche photodiode. The quantum efficiency $\eta$ is represented by the beam splitter (BS). Single arrowheads within the realistic photodetector indicate Poisson processes. For details, see text.

Our aim is to derive the quantum trajectories for the quantum system (the source of the light entering the APD) conditioned on the observation of an avalanche. For the TLA, we consider direct detection of fluorescence. In this case the supersystem is described by the set $\left(\tilde{\rho}_{0}, \tilde{\rho}_{1}, \tilde{\rho}_{2}\right)$, where the tilde indicates that (for simplicity) we are using unnormalized system states, and the subscript indicates the associated detector states. The normalized conditioned TLA state is

$$
\rho_{\mathrm{c}}=\tilde{\rho}_{\mathrm{c}} / \operatorname{Tr}\left[\tilde{\rho}_{\mathrm{c}}\right] ; \quad \tilde{\rho}_{\mathrm{c}}=\tilde{\rho}_{0}+\tilde{\rho}_{1}+\tilde{\rho}_{2} .
$$

Our description of internal dynamics of an APD can be simply translated into rate equations for the discrete detector state $s \in\{0,1,2\}$, which in turn imply the following stochastic generalization of the ME (1):

$$
\begin{aligned}
& d \tilde{\rho}_{0}=d t\left\{\left[\mathcal{L}-\gamma_{\mathrm{dk}}-\eta \Gamma \mathcal{J}-\dot{\mathcal{N}}\right] \tilde{\rho}_{0}+\dot{\mathcal{N}}\left(t^{*}\right) \tilde{\rho}_{2}\right\} \\
& d \tilde{\rho}_{1}=d t\left\{\left[\mathcal{L}-\gamma_{\mathrm{r}}-\dot{\mathcal{N}}\right] \tilde{\rho}_{1}+\eta \Gamma \mathcal{J} \tilde{\rho}_{0}+\gamma_{\mathrm{dk}} \tilde{\rho}_{0}\right\} \\
& d \tilde{\rho}_{2}=d t\left\{\left[\mathcal{L}-\dot{\mathcal{N}}\left(t^{*}\right)\right] \tilde{\rho}_{2}+\dot{\mathcal{N}} \tilde{\rho}_{1}\right\}
\end{aligned}
$$

Here $\mathcal{J} \tilde{\rho}_{0}$ denotes $\sigma \tilde{\rho}_{0} \sigma^{\dagger}$. We use $\mathcal{N}$ for the number of detections counted, so that $d \mathcal{N}(t)=\dot{\mathcal{N}} d t$ is a point process equal to 1 in the infinitesimal interval when an avalanche is first observed and 0 otherwise. The delayed process, $\dot{\mathcal{N}}\left(t^{*}\right) \equiv \dot{\mathcal{N}}\left(t-\tau_{\text {dd }}\right)$, is used to return the de- tector to state 0 . The statistics of $d \mathcal{N}$ are defined by its expectation value $\mathrm{E}[d \mathcal{N}]=\gamma_{\mathrm{r}} d t \operatorname{Tr}\left[\tilde{\rho}_{1}\right] / \operatorname{Tr}\left[\tilde{\rho}_{\mathrm{c}}\right]$.

The detector imperfections lead to substantial changes in the conditional dynamics of the TLA, as compared to ideal quantum trajectories. Representative features can be seen in Fig. 2 (A) and (B). Plot (A) shows a typical portion of a trajectory for $z_{\mathrm{c}}$, while plot (B) shows the same, and $y_{\mathrm{c}}$, over a shorter time around $t \approx 4.9$, when an avalanche is registered. Unlike the case of ideal detection, the corresponding "quantum jump" does not take $z_{\mathrm{c}} \rightarrow-1, y_{\mathrm{c}} \rightarrow 0$, and the amplitude of subsequent oscillations in $z_{\mathrm{c}}, y_{\mathrm{c}}$ are less than 1 . The jumps in the conditioned quantum state caused by the detection of avalanches are attenuated because of the finite detector response time in combination with the continuous Rabi oscillation, which evolves the TLA away from the ground state for a random and unknown time (with mean $\gamma_{\mathrm{r}}^{-1}$ ) between the "actual" spontaneous emission event and the registration of the photocurrent avalanche. During the APD dead time the effective efficiency is zero, and as a result the TLA's conditional state regresses towards the steady state of the unconditional ME (1). Even after the detector becomes ready again (by resetting to state 0 ) the Rabi oscillations in $z_{\mathrm{c}}, y_{\mathrm{c}}$ decay because of the APD's non-unit efficiency and finite bandwidth.

These imperfections cause the stationary ensembleaveraged conditional purity $p=\lim _{t \rightarrow \infty} \mathrm{E}\left\{\operatorname{Tr}\left[\rho_{\mathrm{c}}^{2}(t)\right]\right\}$ to be substantially less than one for large $\Omega$. For small $\Omega$ however, even the unconditional (without measurement) stationary purity $p_{\mathrm{u}}$ of the TLA approaches unity. It is thus useful to define a scaled purity $\in[0,1]$ that measures how much improvement measurement gives: Scaled $p=\left(p-p_{\mathrm{u}}\right) /\left(1-p_{\mathrm{u}}\right)$. For the typical parameter values used in Fig. 2, the Scaled $p \approx 0.052$.
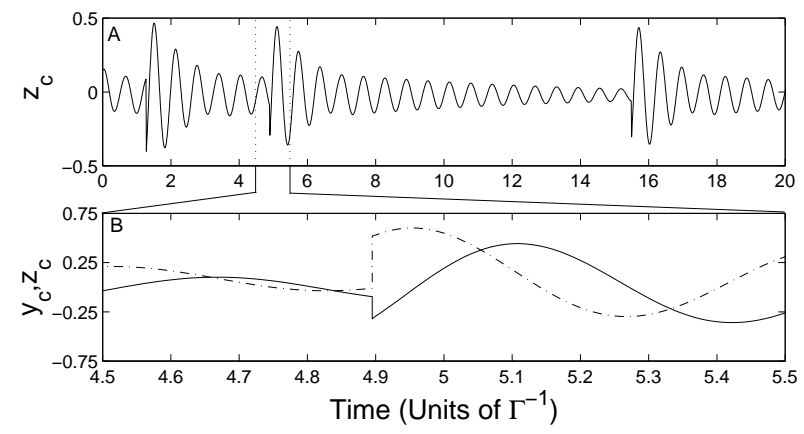

FIG. 2. In plot $(\mathrm{A}), z_{\mathrm{c}}=\operatorname{Tr}\left[\left(2 \sigma^{\dagger} \sigma-1\right) \rho_{\mathrm{c}}\right]$ is shown for a typical trajectory of duration $20 \Gamma^{-1}$. In plot $(\mathrm{B}), z_{\mathrm{c}}$ and $y_{\mathrm{c}}=-i \operatorname{Tr}\left[\left(\sigma-\sigma^{\dagger}\right) \rho_{\mathrm{c}}\right]$ (dash-dot) are shown for the same trajectory near the time of the second APD avalanche. The realistic parameters used for this photodetection trajectory were $\eta=80 \%$, and (in units of the TLA decay rate $\Gamma=300 \mathrm{Ms}^{-1}$ ) $\gamma_{\mathrm{r}}=7, \tau_{\mathrm{dd}}=2, \gamma_{\mathrm{dk}}=5 \times 10^{-6}$ and $\Omega=10$. 


\section{PHOTORECEIVER}

When the incident photon flux is high, as in homodyne detection, a p-i-n photodiode connected to a transimpedance amplifier (see Fig. 3) is an appropriate photoreceiver 17]. When a photon strikes the depletion region of the p-i-n junction, an electron-hole pair is produced, with probability equal to the quantum efficiency $\eta$. The charge carriers drift under the influence of the below-breakdown reverse bias, and the resultant current $I$ is fed into an operational amplifier (op-amp) set up as a transimpedance amplifier. This has a low effective input impedance, so that the diode acts as a current source, and $I$ is converted into a voltage drop $V$ across the feedback resistor, $R$. The capacitor $C$, in parallel with $R$, represents the total capacitance from the output of the op-amp back to its input, including capacitance added deliberately for the smoothing of noise and oscillations. If no electronic noise were present, the output voltage of the photoreceiver would be a filtered version of the input signal given, in the frequency domain, by

$$
V(\omega)=-I R /(1+i \omega R C) .
$$

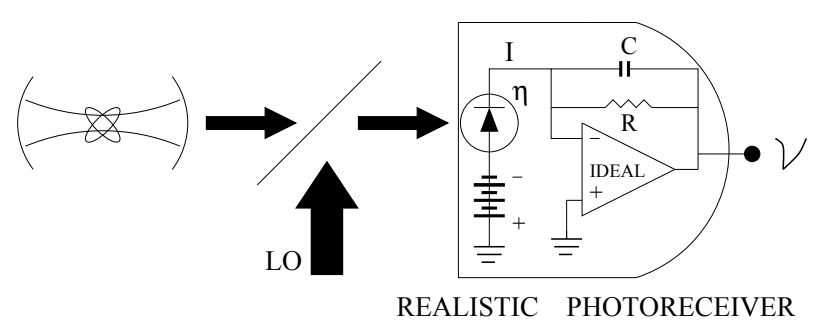

FIG. 3. Homodyne detection by a photoreceiver. The output field of the TLA is combined with a LO before being detected by a realistic photoreceiver consisting of a p-i-n photodiode (of quantum efficiency $\eta$ ) that produces the photocurrent $I$, and an ideal op-amp with feedback resistor $R$, and capacitance, $C$ (see text). The output voltage $\mathcal{V}$ is what is measured in the laboratory.

It should be noted that if this were the case (that is, if there were no noise) then the input $I$ could be perfectly reconstructed from the filtered signal $V$. Thus the resultant quantum trajectories would be no different from those of a photoreceiver with infinite bandwidth. Everything of interest results therefore from the presence of excess noise. We include only the Johnson noise $V_{\mathrm{J}}$ from the feedback resistor, which has a flat spectrum $S_{\mathrm{J}}=4 k_{\mathrm{B}} T R$. This simplification (neglecting contributions from voltage noise of the operational amplifier) can be justified for practical receivers with $R \sim 10 \mathrm{k} \Omega$.

The output voltage $\mathcal{V}$ from the photoreceiver is given by the sum of the filtered signal and the Johnson noise

$$
\mathcal{V}=V+V_{\mathrm{J}}
$$

Our aim is to find the quantum trajectory for the system, conditioned on continuously monitoring $\mathcal{V}$. Since the voltage $V$, which describes the detector state, is a continuous variable, in this case $\mathbf{S}=\mathbb{R}$, the real line, and the supersystem can be described by an operator function $\rho(V)$. Finding the stochastic equation of motion for $\rho(V)$ is quite involved.

We begin by taking the output current $I$ of the photodiode to be that from a perfect (apart from its efficiency $\eta$ ) unbalanced homodyne detection of the fluorescence of the TLA. For a LO tuned to the atomic transition frequency $\omega_{0}$, of power $P$, and phase $\phi$, the current is [6, 19]

$$
I=e \sqrt{P / \hbar \omega_{0}}\left[\eta \sqrt{\Gamma}\left\langle e^{-i \phi} \sigma+e^{i \phi} \sigma^{\dagger}\right\rangle+\sqrt{\eta} \xi(t)\right]
$$

where we have ignored the D.C. component due to the LO power. Here $\xi(t)$ is the Gaussian white noise 18 arising from the Poissonian statistics of the $\mathrm{LO}$ and $e$ is the electron charge. The evolution of the TLA conditioned on $I$ is given, in terms of the noise $\xi(t)$, by the following stochastic master equation 19]

$$
d \rho_{I}=d t\left\{\mathcal{L}+\sqrt{\eta \Gamma} \xi(t) \mathcal{H}\left[e^{-i \phi} \sigma\right]\right\} \rho
$$

where $\mathcal{H}[A] \rho \equiv A \rho+\rho A^{\dagger}-\operatorname{Tr}\left[A \rho+\rho A^{\dagger}\right] \rho$.

Now Eq. (6) is equivalent to the stochastic equation

$$
I+V / R+C(d V / d t)=0 .
$$

Since the voltage $V$ is not directly measured, we must consider a distribution $P(V)$ for it. Assuming that $C>0$, and, for the moment, that $I$ is known, Eq. (10) can be converted to an Itô 18 stochastic Fokker-Planck equation for $P(V)$ conditioned on the photocurrent:

$$
d P_{I}(V)=\left(\frac{\partial}{\partial V} \frac{V+I R}{R C}+\frac{P \eta e^{2}}{2 \hbar \omega_{0} C^{2}} \frac{\partial^{2}}{\partial V^{2}}\right) P(V) d t .
$$

Here we are using the convention that subscripts indicate that the increment is conditioned on that result. That is, for example, $P_{I}(V) \equiv P(V \mid I)$.

Next we need to determine the effect of the measurement of $\mathcal{V}$ on $P(V)$. This can be calculated by using Bayes' conditional probability theorem

$$
P_{\mathcal{V}}(V)=P_{V}(\mathcal{V}) P(V) / P(\mathcal{V})
$$

Remembering that the Johnson noise is white, it follows from Eq. (7) that $P_{V}(\mathcal{V})$ is a Gaussian with mean $V$ and variance $4 k_{\mathrm{B}} T R / d t$. From this we find that

$$
P(\mathcal{V})=\int d V P_{V}(\mathcal{V}) P(V)
$$

is a Gaussian of mean $\langle V\rangle$ and variance $4 k_{\mathrm{B}} T R / d t$. It follows that we can write

$$
\mathcal{V}=\langle V\rangle+\sqrt{4 k_{\mathrm{B}} T R} d W_{\mathrm{J}}(t) / d t
$$


where $d W_{\mathrm{J}}(t) / d t$ is another Gaussian white noise source, independent of $\xi(t)$. Substitution of $P_{V}(\mathcal{V})$ and $P(\mathcal{V})$ into Eq. (12) yields the effect of the $\mathcal{V}$-measurement:

$$
d P_{\mathcal{V}}(V)=(V-\langle V\rangle) P(V) d W_{\mathrm{J}}(t) / \sqrt{4 k_{\mathrm{B}} T R} .
$$

Now, to see how $\mathcal{V}$ conditions the TLA, we form the quantity $\rho(V)=\rho P(V)$, where $\rho$ is here independent of $P(V)$ because we are imagining $I$ to be known at all times. The time evolution of $\rho(V)$, given that $\mathcal{V}$ and $I$ are known, is found from

$$
\begin{aligned}
\rho(V)+d \rho_{I, \mathcal{V}}(V)= & \left(\rho+d \rho_{I}\right) \\
& \times\left[P(V)+d P_{I}(V)+d P_{\mathcal{V}}(V)\right],
\end{aligned}
$$

with the use of Eq. (11), Eq. (15) and Eq. (9).

Finally, in reality, $\mathcal{V}$ is known but $I$ is not. Therefore we should average over the vacuum noise $\xi(t)$, but keep the Johnson noise $d W_{\mathrm{J}} / d t$. We define a dimensionless voltage $v=V \sqrt{C / 4 k_{\mathrm{B}} T}$, a rate $\gamma=1 / R C$ and a dimensionless noise power $N=4 k_{\mathrm{B}} T \hbar \omega_{0} / \eta R P e^{2}$. This last expression is the ratio of the low-frequency power in $\mathcal{V}$ from the Johnson noise to that from the vacuum noise. We then obtain the following stochastic nonlinear superoperator Fokker-Planck equation for $\rho(v)$ :

$$
\begin{aligned}
d \rho_{\mathcal{V}}(v)= & d t\left(\mathcal{L}+\frac{\gamma}{2 N} \frac{\partial^{2}}{\partial v^{2}}+\gamma \frac{\partial}{\partial v} v\right) \rho(v) \\
& +d t \frac{\partial}{\partial v} \sqrt{\frac{\gamma \Gamma \eta}{N}}\left[e^{-i \phi} \sigma \rho(v)+e^{i \phi} \rho(v) \sigma^{\dagger}\right] \\
& +\sqrt{\gamma} d W_{\mathbf{J}}(t)(v-\langle v\rangle) \rho(v) .
\end{aligned}
$$

The dependence on $\mathcal{V}$ may be explicated by substituting $d t \gamma\left(\sqrt{C / 4 k_{\mathrm{B}} T} \mathcal{V}-\langle v\rangle\right)$ for $\sqrt{\gamma} d W_{\mathrm{J}}(t)$ [see Eq. (14)]. In the above, $\langle v\rangle=\int d v \operatorname{Tr}[\rho(v)] v$. The normalized conditioned TLA state is $\rho_{\mathrm{c}}=\int \rho(v) d v$.

A Typical trajectory for realistic homodyne $x(\phi=0)$ detection is shown in Fig. A (A). The main difference from the case of perfect detection is the reduced amplitude of variation in $x_{\mathrm{c}}$ (dotted) and $z_{\mathrm{c}}$ (solid). This is due to the effective bandwidth of the photoreceiver, which affects $z_{\mathrm{c}}$ more because of its faster dynamics. Plot (B) shows the photoreceiver output voltage $\mathcal{V}$ that is used in Eq. (17) to condition the TLA state. It is seen that $\mathcal{V}$ is correlated with $x_{\mathrm{c}}$ as expected.

\section{See attached file TrajHom.jpg}

FIG. 4. Plot (A) shows $x_{\mathrm{c}}=\operatorname{Tr}\left[\left(\sigma+\sigma^{\dagger}\right) \rho_{\mathrm{c}}\right]$ (dotted) and $z_{\mathrm{c}}$ (solid) for a realistic homodyne $x$ trajectory. Plot (B) is the dimensionless output voltage from the photoreceiver. The photoreceiver parameters were $N=0.1, \eta=98 \%, \gamma=1.5 \Gamma$. System parameters were as for Fig. 2. Plot C gives the Scaled $p$ as a function of the driving, $\Omega$, for homodyne $x$ (dotted) and $y$ (dash-dot) detection.
Plotted in (C) is the scaled purity as a function of the driving strength for both homodyne $x$ and $y(\phi=\pi / 2)$ detection. As $\Omega$ increases, homodyne $y$ detection becomes increasingly worse than $x$ detection at following the evolution of the TLA. This is due to the finite bandwidth of the photoreceiver in combination with the conditional homodyne dynamics in the $\Omega \gg \Gamma$ limit [7]. For homodyne $x$ measurement the $x$ quadrature, which changes sign fairly infrequently, dominates the TLA state. The slow $(\Gamma)$ dynamics allow the detector to track of the state reasonably well. In contrast, homodyne $y$ detection produces a conditional state dominated by fast $(\Omega)$ Rabi cycling, which is poorly followed.

\section{CONCLUSIONS}

In conclusion, we have presented a theory of quantum trajectories for systems conditioned on realistic photodetection. The equations are tractable, as we have demonstrated by numerical simulations, and allow us to quantify the degree and manner by which imperfections such as a finite bandwidth modify the conditioning of quantum states by measurement in concrete experimental scenarios. Realistic quantum trajectory models will be of paramount importance in the field of real-time quantum feedback control 20]. The techniques we introduce here may also prove essential in describing other realistic measurements, such as in condensed matter systems [8,13.

[1] J. von Neumann, Mathematical Foundations of Quantum Mechanics (Springer, Berlin, 1932); English translation (Princeton University Press, Princeton, 1955).

[2] E. B. Davies, Quantum Theory of Open Systems (Academic Press, London, 1976).

[3] K. Kraus, States, Effects, and Operations: Fundamental Notions of Quantum Theory (Springer, Berlin, 1983).

[4] J. Dalibard, Y. Castin and K. Mølmer, Phys. Rev. Lett. 68, 580 (1992).

[5] C. W. Gardiner, A. S. Parkins, and P. Zoller, Phys. Rev. A 46, 4363 (1992).

[6] H. J. Carmichael, An Open Systems Approach to Quantum Optics (Springer, Berlin, 1993).

[7] H. M. Wiseman and G. J. Milburn, Phys. Rev. A 47, 1652 (1993).

[8] H. M. Wiseman et al., Phys. Rev. B 63, 235308 (2001)

[9] J. Gambetta and H. M. Wiseman, to appear in Phys. Rev. A (2001).

[10] J.-I. Cirac, P. Zoller, H. J. Kimble, and H. Mabuchi, Phys. Rev. Lett. 78, 3221 (1997).

[11] A. C. Doherty and K. Jacobs, Phys. Rev. A 60, 2700 (1999).

[12] H. Mabuchi, J. Ye, and H. J. Kimble, Appl. Phys. B 68, 1095 (1999).

[13] M. H. Devoret and R. J. Schoelkopf, Nature 406, 1039 (2000). 
[14] P. R. Rice and H. J. Carmichael, IEEE J. Quantum Elect. 24, 1351 (1988); Q. A. Turchette, R. J. Thompson, and H. J. Kimble, Appl. Phys. B 60, S1 (1995).

[15] B. T. Debney and A. C. Carter, Optical Fiber Sensors Components and Subsystems, Ch. 4, Vol. 1 (Artech House, Boston 1996).

[16] B. Garside, Optical Fiber Sensors Components and Subsystems, Ch. 5, Vol. 3 (Artech House, Boston 1996).
[17] J. D. C. Jones, Optical Fiber Sensor Technology, Ch. 4 (Chapman and Hill, London 1995).

[18] C. W. Gardiner, Handbook of Stochastic Methods (Springer, Berlin, 1985)

[19] H. M. Wiseman and G. J. Milburn, Phys. Rev. A 47, 642 (1993).

[20] A. C. Doherty et al., Phys. Rev. A 62, 012105 (2000). 
This figure "TrajHom.jpg" is available in "jpg" format from: http://arXiv.org/ps/quant-ph/0112129v1 